\title{
Johann Bizer/Alexander Roßnagel Sicherheit in der Informationstechnik - Aufgabe für ein neues Bundesamt
}

\section{Die Verletzlichkeit der „Informationsgesellschaftex}

Seir dem r. Januar r 99 r gibt es das Bundesamt für die Sicherheit in der Informationstechnik (BSI). Das Amt soll mit 270 Mitarbeitern die Sicherheit in der Informationsteclinik erhöhen. "Viele Bereiche von Wirischaft und Verwaltung sind bereits heutc von dem einwandfreien Funksionieren der Informationstechnik abhängig. Mit dem zunehmenden Einsatz der Informationstechnik steigen auch die damit verbundenen Risiken durch unrichtige, unbefugt gesteuerte, fehlende oder rechtsgutgefährdende Informacionen. $"{ }^{\prime}$

Knapp, aber zutreffend beschreibt die Bundesregicrung mit diesen Worten die steigende Verletzlichkeit dor modernen Informationsgesellschaft $\alpha^{2}$ und damit das Problem, für das das neue Amt dic Lösung sein soll. In der Tat werden Fehler der Informations- und Kommunikationstcchnik, Fehlbedienungen und böswillige Mißbrauchshandlungen erheblich bedrohlicher, wenn die Abhängigkeit der Gesellschaft vom Funktionieren der automatischen Informationsverarbeitung und -übermitclung und damit das potenticlle Schadenspotential gegenüber heute noch beträchclich ansteigen werden. Dem Bild der anformationsgesellschaft entsprechend, das man hierzulande hoffnungs-3 oder besorgnisvoll' zeichnet, werden Informationsverarbeitung und Telckommunikation in Umfang, Verbreitung und Bedeunng zunchmen, noch stärker in die Gesellschaft eindringen und zu einem vernetzten System zusammenwachsen. Dabei werden sie andere Formen der Informationssammlung, -verarbeizung und Kommunikation verdrängen und schließlich weitgehend ohne Alternative und Substitutionsmöglichkeit sein. Störungen in einem Bercich werden sich dann schnell auf andere Bereiche übertragen. Eine IuK-gestürzte Warenwirtschaft wird auf der völligen Vernetzung von Lieferanten, Zulieferem, Produzenten, Händlern, Kunden und Banken beruhen. Vom Funktionieren der luK-Systeme werden auch die Energieversorgung, die medizinische Versorgung, das gesamte Zahlungssystem, die wichtigsten Dienstleistungen, wissenschaftliche Organisationen, das Verkehrssystem, die Medien, der Umweltschutz sowie die stantliche Vervaltung und die politische Steverung abhängig sein.s

I Einlcrung zur amelichen Begründung des BSIG, BR-Drs. 134/80, S. $1=$ BT-Drs. $11 / 7029$.

2 BR-Drs. 134/90, S. x; s. auch Bundesınnenministcr Schäuble, BT-Sten.Ber. 11/16794; Stantssekrecär Neuscl, Akuvitaten der Bundesregierung zur IT-Sicherher, Vorirag aul der i. Dcuischen Konferenz über Computersicherhext 15./16. Mai 1990, ROV 1990, S. 16rfí.

3 S. z. B. Bundesminıseer für Forschung und Technologıe/Bundesmenister fur W/rtschast. Zukunftskonzept Informationstechnik, Bonn 1989 .

4 S. z.B. Kubıcek/Rolf, Mikropolis, Hamburg 2. Aufl. 1986; Roßnagel/Wedde/Hammer/Pordesch, Vighcalisıerung der Grưfdrechte. Zur Verfassungsverträglichkeıt der Informatıons- und Kommunikatıonstechnik. Opladen 1990.

s S. hicrzu náher das s Zukunftsbilde In Roßragcl/Wedde/Hammer/Pordesch, Die Verickalichkele der Informationsgescillschafle, Opladen 2. Auf. 1990,18 f́. 
Bereits in der Vergangenheit haben eine Vielzahl von Vorfälen die Verlctzlichkeit der "Informarionsgesellschaft" angedeutet. So fielen beispielsweise am 21 . Januar 1990 in den USA alle : 14 Fernvermitclungsstellen im landesweiten Telekommunikationsnerz von AT \& T gleichzcirig aus. Ein identischer Softwarefehler im Routingsystem führte für neun Stunden zu einer Überlastung aller Vermittlungscomputer. Schäczungsweise 75 Millionen Telekommunikationsverbindungen konnten durch diesen Ausfall nicht vermittelt werden. Durch einen Brand in der Vermittlungszentrale der Illinois Bell Telephone Company wurden 1988 Tausende von Unternchmen und Haushalten für Wochen von jeder Telekommunikarion abgeschnicten. $19 \$ 7$ gingen „Oldsmobil « die kompletien Designdaten eines neuen Automodells durch einen Computerfehler verloren. Bundesdeutschen Hackern gelang es im September 1987, über Fehler im Betriebssystem zweier NASA-Rechncr das Space Physics Analysis Nerwork zu erreichen und von diesem aus in 136 Computer cinzudringen, dic Daten sowohl aus zivilen als auch militärischen Projekten verarbciteten. Im Herbst 1988 legte ein Computervirus, den ein Student in zwei Computernetze implanciert harte, über 6000 Rechnex lahm und zerstörte etliche tausend Daceien und Programme in der US-Bundesverwaltung.

Vorfälle dieser Art ${ }^{6}$ gefährden den Weg in die rInformationsgesellschaft $\alpha$. Um ihrer Verletzlichkeit entgegen zu wirken, hat der Bundestag am 24. Okzober 1990 das " Gesecz über die Errichrung des Bundesamts für die Sicherheir in der Informationstechnik (BSIG) verabschieder.7 Im folgenden werden Geschichte des BSI (2.) und der Inhalt scines Errichtungsgesetzes (3.) kurz beschrieben, das Amt von seinen Aufgaben und Befugnissen her kritisch beleuchter (4. und 5 .) und an notwendigen und möglichen Alternativen gemessen (6.).

\section{Vorgeschichte des $B S I$}

Aufbau und Konzept des BSI scützen sich auf die in den joer Jahren gegründere Zentralscelle für das Chiffricrwesen $(\mathrm{ZFCH}) .{ }^{8}$ Die $\mathrm{ZFCH}$ war dem Bundesnachrichtendienst (BND) 9 zugeordnet, unterstand dem für die Koordination der Geheimdienste zuständigen Staatssekretär des Bundeskanzleramces und zählte damit zum Geschäfisbereich des Bundeskanzlers. Ihre Aufgabe war neben dem Ver- und Eneschlüsseln von Nachrichten der eigenen "Dienste* auch dic Sicherung der Kommunikation der Bundcsverwaltung - wie beispieisweise des Auswärtigen Amtes mit den eigenen Botschaften im Ausland - sowie das Entschlüsseln von Nachrichten, die im Rahmen der Auslandsaufklärung und Spionageabwehr abgefangen oder abgehört worden sind. ${ }^{10}$ Anfang der joer Jahre wurde der $\mathrm{ZfCH}$ die Überprüfung der für die Verarbeitung oder Übertragung von Vcrschlußsachen eingesetzten Compurerhardware auf die Abstrahlsicherheir übertragen. 1987 wurden die Aufgaben der $\mathrm{ZfCH}$ um den Bereich "Computersicherheic e erweiter."

\footnotetext{
6 S. t.u ihnen und vicken anderen Roßnagul/Wodde/Hammes/Pordesch (Fn. s), 69ll., 106 ff., 129 If. sowic den Berichı der Bundesrechnungshofs BT-Drs. $11 / 7691$.

7 S. BT-Sien. Ber. $11 / 18247 f 1$.

8 Zur Geschichte siche auch das Hintergrundpapicr von Statusckretär Hans Neusel auf der Wissenschafespressckonlereriz vom 6. 2.1990 sawic ders. (Fn. 2), S. $16_{4}$ If

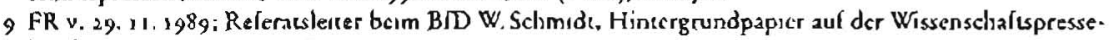
konfercnz vom 6.2.1990. S. 2 -

10 Neusel (Fn.8), S.1: Kerseen, 6.RDV-Forum der DAFTA, 13. Tagung der DAFTA arn 16. und 17.1 1.89; FR v. 29.11.89; s. hierzu auch die VS-Fernmelderschulinien.

11 Neusel (Fn. \$), S. 2; ders. (Fn. 2) S. 164 S, s. zur Guschıchıe der ZSL und alınlichen Entwicklungen un den USA, dic zu victen Belürchtungen un der kritıschen Bcurtcilung dieses Gesctzesvorhabens geführt haben,
} 
Ihre politischen Handlungsabsichten in diesem Feld hat die Bundesregierung in ihrem am 23. November 1989 gebilligten »Rahmenkonzept zur Gewährleistung der Sicherheit bei Anwendung der Informationstechnik (IT) zusammengestellt. ${ }^{2}$ Die Errichtung des neuen Bundesamts ist das "Kernscück im Rahmen dieses Handlungskonzeprs. "3 Der Geseczentwurf ist daher im Kontext der Zielserzungen und Handlungsvorschläge dieses Rahmenkonzepts zu beurteilen.

Mit der zunehmenden Aufgabenerweiterung im Bercich Computersicherheit wurde die ZfCH zum r. Juni 1989 in "Zentralstelle für Sicherheit in der Informationstechnik " (ZSI) umbenannt. Sie befand sich wciterhin im Geschäftsbereich des Bundeskanzleramtes. ${ }^{14}$ Thre Aufgaben wurden in dem Rahmenkonzept der Bundesregierung nun aber über den Bereich des staatlichen Geheimschutzes hinaus auch auf Sicherheitsaspekte der sivilen Anwendung der Informationstechnik ausgedelnnt. ${ }^{\text {s' }}$

Mit dem BSIG wurde nun die ZSI in eine Bundesoberbehörde umgewandelt. Sie soll allerdings nicht mehr dem Bundeskanzleramt zugeordnet sein, sondern als wzivile


bereich Entzifferung « soll beim Bundeskanzleramt und damit beim BND verbleiben. ${ }^{18}$ Damit ist das BSI zumindest organisatorisch aus dem Gehcimáienstbercich herausgenommen. Allerdings besteht nun mit der Zuordnung zum Bundesminister des Innern (BMI) eine Nähe zu den Sicherheitsbehörden des Bundes, insbesondere dem Bundesame für Verfassungsschurz und dem Bundeskriminalamt. '9 Personelle Bezüge werden aufgrund des Mangels an qualifiziertem Personal auch zur alten $\mathrm{ZfCH}$ bestehen. ${ }^{20}$ Dor Leiter des BSI ist der langjährige Leiter der ZfCH und der ZSI. Der Gründungsstamm von 153 Mitarbeitern wird aus dem BND übernommen. Bis 1994 soll sich dann dic Zahl der Mitarbeiter auf 270 erhöhen."

Die parlamencarischen Beratungen verliefen ohne besondere Komplikacionen. Der Bundesrat empfahl in seiner Stellungnahme im wesentichen nur Änderungen zu den beamtenrechtlichen Regelungen. Ansonsten schlug er vor, auch Länderbchörden von dem künftigen Sicherheitswesen des BSI proficieren zu kassen. ${ }^{22}$ Die SPD unterstutzte die Errichtung des neuen Bundesamtes, kritisierte jedoch die Ressortie-

auch Bizer/Hammer/Pordesch/Roßnagel, Das ncue Bundesami fur Sichcrhct in der Informationstechnik. Planungen - Kritik - Vorschlage. Prover-Projekibercicht 4. Darmstade Februar 1990, jif., 17 ff.

12 Zit. als IT-Sicherhellsrahmenkonzcpe. Eine vorläufige Version dieses Rahmenkonzepus vom 27.9. 1989 wurde in DuD 1989, S. 19r ff. veröffentlicht.

if S. Neusel (Fn. 2), S. 165 .

if Vgl. Prïsident der ZSI O. Leibench, Hintengrundpapier aul der Wissenschafispressekonleren\& vom 6. 2. 1990, S. I.

is Die Aufgaben der ZSI bis zum Erlaß cincs Ernchungsgesetzes ergeben sich aus Aniage 3 des Sichcrheitsrahmenkonzeptes, DuD 1989, S. 297 f. Zur Zeu iverden von der ZSI ca. 10 IuK-Sysecme evaluier, en IT-Evaluationshandbuch erseelle und Vorarbenen für ein IT-Sicherlsetı shandbuch geleistet - Kersicn, 13 DAFTA, 1989; Neusel (Fn. 2), S. 16 lf. Ein nanonaler Kriterienkatalog zur Evaluanon von

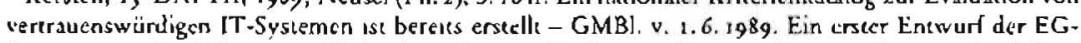
Kommission der Information Technology Security Evalunion Criteria, der unier Leisung der ZSI in einem internationalen Arbeitskerss crerbette worden sst, liegt ebentalls berests vor - Version or vom 2.3. 1990, hrsg. vom Bundesinncriminisicrium.

16 Neusel (Fn. 2), S. 165 .

17 BR-Drs. x 34/90, S. 10; vgl. schon IT-Sicherheitxrahmenkonzepl, Pkt. 9.5. I. Die Facliauisıcht wird von enem speziellen Referat is Sicherhese in der Informalonsecrhnike sowve der Koordinicrungs- und Buesungsstelle der Bundesregierung in der Bundesverwaltung im BMI (KBSt) ausgeübt werden, vgl. N cusel $\left(\Gamma_{n}, 2\right)$.

18 Neusel (Fn, 2)

19 Beide untersichen dem BMl, vgl. Is Abs, 2 Nr, 2 und Abs. 3 Nr, 2 BKAG; $\$ 2$ Abs, 1 BVerlSchG.

20 BR.Drs. $134 / 90$, S. 2.

21 BR-Drs. 134/90, S. 3. Inwieweil der Mangel an Kryptographen durch Übcmahme chernaliger Mirarbesier des Zentralen Chiffrucrorgans der DDR gedeckr wird, ist noch ungewiß - s. Abg. Such, BT-Sten. Ber. $11 / 18252$.

22 BR-Drs. $134 / 90$ (BeschluB). 
rung des Bundesamts beim Bundesinnenminister, fehlende Hafrungsregelungen und den rein rechnischen Sicherheitsbegriff des Geseczes. Außerdem monierte sie, daß eine Technikfolgenabschätzung und -bewenung der künftigen Risikopotenciale im Aufgabenkatalog des Ames fehle. ${ }^{23}$ Die GRÜNEN machren darüberhinaus rechtiche Bedenken gegen die Zusammenarbeit zwischen BSI einerscits und Bundeskriminalamt, Verlassungsschure und Gehcimdiensten andererseirs geltend und kritisicrten die Aufgaben- und Befugniszuweisung als probleminadäquar. ${ }^{4}$ Sie legten einc Fülle von Änderungsvorschlägen vor, die das Ziel verfolgren, dem Amt einen unabhängigen Stacus zu verschaffen, seinc Zielserzungen auf die Gewährleiscung von Bürgersicherheit zu konzentrieren und ihm Befugnisse einzuräumen, eine Reduzienung der Verletzlichkcit der Gesellschaft auch tacsächlich zu erreichen. ${ }^{25}$ Die Vorschläge der Opposition fanden inhaltlich ihren Niederschlag in der Stellungnahme des Ausschusses für Forschung, Technologie und Technikfolgenabschärzung, wurden aber vom fcóerführenden Innenausschuß zurückgewiesen. ${ }^{26}$

In der Offentlichkeit stieß das Gesetzesvorhaben auf wenig Interesse. ${ }^{27}$ Dic Auseinanderseczungen beschränkten sich vorwiegend auł Fachkongresse ${ }^{28}$ und die Fachliteratur ${ }^{29}$. Kricische Stellungnahmen wurden vom Deutschen Gewcrkschafrsbund ${ }^{30}$, dem Forum der Informatiker und Informarikerinnen für Frieden und gesellschaftliche Verantwortung (FiFF) ${ }^{\prime 1}$ und der Gescllschaft für Informatik (GI) ${ }^{32}$ vorgelegt. Die Kritik wurde - in unterschiedlicher Stringenz - von der Opposition aufgegriffen und in die parlamentarischen Beratungen eingelührt. Dadurch wurde crreicht, daß die Aufgaben des BSI um die Technikfolgenabschätzung erweiter ${ }^{33}$ und die Beratungspflichten für BKA und Veriassungsschurz präzisierr wurden.

23 S. der Abg. Patcrna, BT-Stcn.Ber. 11/1679s f. und 18248 ff.; dic auf enc SPD-Inutuave zuruickgehende Stellungnahme des Ausschusses für Forschung, Technologre und TechnikJolgenabsehaityung des Bundestages zum Eniwuris des BSIG vom 12.9. 1990-BT-Drs. 11/8177, 10 ff.

24 S. die Abg. Rust, BT-Sien.Ber. 11/167961; Abg. Such, BT-Sten. Ber. 11/182516.

25 S. BT-Drs. 11/7246; $11 / 8177$ und $11 / 8197$.

26 S. zu beidon BT-Drs. 11/\$177.

27 Eine Ausnahme bildet der Arcikel von Gunhild Luige, Alles unter Kontrolle? in der Zent Nr. 20 vom 11. S. 1990.

28 Z. B. dic 2. Deuesche Konkerenz über Computersicherheit in Bad Godesberg 2m is.116. 5. 1990 oder die Tagung "Zukunftskonzepi Infonnationstechnik a der Gesellschaft für Informalik vom 14.-17.6. 1992.

29 Bizer/Hammer/Pordesch/Roßnagel, Ein Bundesame für dic Sicherhet un der Infarmstionstechnik Krilsche Bernerkungen zum Gesetzentivurf der Bundesregjening. DuD 1990, 178 ff.; Bernharde/ Ruhmann, Wie en Geheımdiense zur oberseen Bundesbehörde fur Computersıcherhent gemacht wird, FiFF-Kommunikation 2/go, 29ff.; Roßnagcl, BSI: Kein BeIIrag zur Vemngerung der Verleczliclikeıt. Computerwoche 12 vom 23-3-1990, 8: Kersecn, ZSI/BSI: Eine statliche Inieıatuve sur I'T-Sicherheı, Compurerwache 12 vom 23-3-1990, 40 ff.; Bernbard/Ruhmann, Nulation einer Geheimdienststelle, Computenwache 12 vom 23.3.1990, 44 ff.: Schaublc, Wider Knmınaliat und Akzeptanzverlust, Sieg Tech 4/90, 18 lf.; Beth, Zur Sicherheir der Informationstcchnik, Informatik-Spekerum 1990, S. 104 fi.: Wormann, Konzepic der Bundesregienng zur Sicherheri in der lnlormationstechnik. DuD 1990, S. 453 ff.

30 Richerc, Neuc -Philosophre" muß her, Sieg Tech 4/g9. ir ff.

31 FifF-Kommunikxtion 1/1990, S.8; Compuecrwoche vom 22. 12. 1989, S.6.

32 Stellungnahme der Arbeisgruppe 10 des FB 8 der GI zum Entwurf des Emchtungsgesctzes dec Bundssregienung für en Bundesame fur Sicherhelt il der informauonstechnik auf der GI-Fachtagung Informacik und Gescllschaft. vom 14. bis 17.6.1990.

33 S. hierzu bereies Bundesinnenminıster Schäublc in der ersecn Lesung im Bundestag am 31. 5. 1990. BT. Sten.Ber. 11/16794 sow,e Neusel (Fn. 2), \$. $16_{4}$ if. Diese Aufybe wurde nach $\$ 3$ Abs. I Nr. 7 BSIG allerdings auf dic Beracung der Hersceller, Verreiber und Anwender beschränkt. 
Als Errichtungsgesecz beschränkt sich das BSIG in $\$$ I auf die Schaffung ciner neuen Bundesoberbehörde. Eine Ziél- oder Zweckbestimmung fehlt." \$ $\$$ beschrànkt sich auf eine Begriffsbestimmung der Informationsechnik als walle wechnischen Mirtel zur Verarbeitung oder Übertragung von Informacionen * und auf eine Definition der * Sicherheit in der Informationstechnik «. Unter diesem Begriff versteht der Gesetzentwurf dic

"Einhaltung bestimmter Sicherheitsstandards, die die Verfügbarkeit, Unversehrheit oder Vertraulichkeit von Informationen betreffen, durch Sicherheitsvorkehrungen $r$ in informationstechnischen Systemen oder

2. Komponenten oder bei der Anwendung dieser Systeme und Komponentena $(\$ 2$ Abs. 2).

Es folgt in $\$ 3$ Abs. I ein umfangreicher Karalog der Aufgaben des BSI, der Forschungs- und Entwicklungsaufgaben beschreibr (Nr. I und 2), die Erteilung von Sicherheitszertifikaten vorsieht (N..3), dem BSI die Zulassung von informationstechnischen Produkıen für den Geheimschuezbereich überirägt (Nr.4) und es zur Untcrstürzung andercr Behörden verpflichtet ( $\mathrm{Nr}$. S bis 7 ). $\$ 4$ regelt das Verfahren und die Voraussetzungen der Zerrifikatsertcilung und $\$ s$ enthält cine Verordnungsermächtigung für den Bundesminister des Innern. In $\$ \$ 6$ bis $6 c$ folgen schließlich beamtenrechtliche Vorschriften.

\section{Staats- und Marktsicherheit statt Bürgersicherheit}

Wer das Geserz mit der Problembeschreibung sciner Begründung vergleicht, dem fällt vor allem auf, daß das Problem der "Verletzlichkeit der modernen Informationsgesellschaft* in der Aufgabenbeschreibung des Amtes auf die Unterscützung der deutschen IuK-Industrie und Hilfestellungen zur Gewährleistung der inneren Sicherheir reduziert wird. Befugnisse zur Verringerung der Verletzlichkeit fehlen völlig.

Vielmehr soll dic deutsche $I$ uK-Industrie unterstützt werden durch die Enrwicklung von Kriterien, Verfahren und Werkzeugen für die Prüfung und Bcwertung der Sicherheit von informationstechnischen Systemen und Komponenten, die Prüfung und Bewertung von ruK-Produkten sowie die Erteilung von Sicherheirszertifikaten ( $\$ 3$ Abs. I, Nr. 2-4). Die Sicherheit in der Informationstechnik soll dadurch erhöht werden, daß die Nachfrage nach nsicheren "IuK-Produkten durch den amclichen Nachweis von Sicherheirsstandards erleichtert wird. Betreibern, Anwendern und Nutzern von Informations- und Kommunikationstechniken sollen die Zertifikate Oriencierungshilfen für die Sicherheitsqualität bestimmter Produkce liefern. Allerdings ist für kein IuK-Produkt - vom PC bis zum Großrechner - der Nachweis der Sicherheit als Zulassungsvoraussetzung vorgeschrieben. Über die sozial gewünschte Sicherheit in der Informationstechnik wird allein der Markt entscheiden.

Auch strebe das BSI für die Zerrifizierung keine Monopolstellung an. Vielmehr soll es jedem frei stchen, ob er das Zertifikat einer anderen Institution dem des BSI vorzieht." Die Marktorientierung des Geseczes zeigt sich auch darin, daß Prüfung

34 Alle Paragraphen ohne Geselzes angaben sund solche des Entwuris emes "Gescexes über die Ernchiung des Bundesames für Sicherheil in der Iniormacionstechnologic (BSI) .

is Neusel (Fn. 8), S. 4; Leibench (Fn. 15), S. 2. 
und Bewertung auch von anderen wsachverständigen Stellen ", die vom Bundesamt hierzu beauftragt werden ( $\$$ \& Abs. 2 ), vorgenommen und daß Sicherheitszertifikate anderer anerkannter Prüfstellen aus dem Bereich der Europäischen Gemeinschaft bei gleichwertiger Sicherheit anerkannt werden können (\$4 Abs. 4).

Durch eigene Sicherlyeitsstandards und die Erceilung von Sicherheitszercifikaten soll insbesondere gegenüber dem US-Markt, der eine eigene behördliche Zerifizierung kennr, die Exportfähigkcit der bundesdeutschen Induscric gesichert werden. ${ }^{16}$ Um eine incernationale Anerkennung der deusschen Zerrisikate zu erreichen, wird eine Abstimmung mic curopäischen bzw. internationalen Kriterien zur Bcwertung, Prüfung und Zercifizierung von IT-Systemen und Komponenten angestrebt, die auch mit der NATO abgestimmt werden sollen." Die ausschließliche Orientierung auf den nationalen und internationalen Markt verengt dic ursprüngliche Zielsetzung des Geserzes, dic "Verletzlichkeit der modemen Informationsgesellschaft" zu verringern, auf die Sicherung der internationalen Wettberverbsfähigkeit.

Für den Bereıch der Inneren Sicherheut soll das Bundesamt aus dem Dunkel seiner Vorgänger, den Geheimdiensten, heraustreten und als technische Fachbehörde die scaatichen Stellen, ninsbesondere soweir sie Beratungs- und Kontrollaufgaben wahrnehmen $(\$ 3$ Abs. I Nr. $\varsigma$ ), unterstützen. Ausdrücklich nennt der Gesetzentwurf den Bundesbeaufreagten für den Datenschutz, adessen Unterstützung im Rahmen der Unabhängigkeit erfolgt, dic ihm bei der Erfüllung seiner Aufgaben nach dem Bundesdatenschutzgesetz zustehisa. Erst aus der Begrundung ist zu entnehmen, daß hicrzu auch dic Koordinierungs- und Beratungsstelle der Bundesregierung für Informationstechnik in der Bundesverwaltung im Bundesinnenministerium (KBSt) sowie vor allem das Bundesame für Vcrfassungsschutz und der Militärische Abschirmdienst sowie das Bundesministerium für Wirtschaft, sorveit es Unternehmen mit Verschlußsachen-Aufträgen des Bundes betreuc, gezählt werden. ${ }^{38}$

Neben dem Geheimschutz erstreckt sich der Aufgabenbereich des BSI aber auch auf die Unterstützung „der Polizei und der Strafverfolgungsbehörden bei der Wahrnehmung ihrer gesetzlichen Aufgaben " sowic der Verfassungsschutzbehörden bei ihrer Tätigkeit. ${ }^{39}$ Das BSI soll seine Fachkenntnisse aus der Sicherung der Informarionsund Kommunikationsdienste zur Analyse, Bewercung und Beweisführung von wallgemein kriminell(en), extremistisch(en) oder nachrichtendienstlich motivierc(n) Einbrüche(n) in informacionstechnische Systeme "den genannten Sicherheitsbehörden zur Verfügung stellen $4^{\circ}$ und wird damic unmitrelbar im Zusammenhang mit Grundrechtseingriffen tätig. Um die Kontrolle des BSI zu erleichtern, hat es die Unterstützungsersuchen aktenkundig zu machen.

Auf die rechtiche Fragwürdigkeit dieser Regclungen ist schon an anderer Stelle hingewiesen worden. "Hervorzuheben ist hier die Konsequenz dieser Verquickung widerstreitender Intcressen. Das BSI kann nicht, wenn es seine Zielvorgabe adäquat erfüllen will, Diener zweier Herren sein. Nach dem BSIG soll es auf der einen Seite

36 BR-Drs. 194/90. S. 2 ; IT-Sicherhestsrahmenkonzept, Pkt. 1.2; 3.1: nacls $\$$ Abs. 2 bedürfen die Entseherdungen des BSI uber Kritersen und Verfahren der Zerifikatseneilung des Einvernehmens mit dem Bundesrounster für Wirschaft.

$37 \$ 1$ Abs. 4; BR-Drs. 134/90, S. 29. IT.Sichcrhestsrahmenkonzepı, Pkt. 2.1; 9.8.1; 9.8.2; 9.8. y; Zur NATO: Pkt, 6.2; $s ; 9.1 ; 9.7$. Zum Veriahren der Zerifikatscrecilung s. ZSI, IT-Evaluationshandbuch, Bonn 1990, S. 68 il.

38 BR-Drs. $134 / 90$, S. 22.

39 Engegen dem Wordaur der Vorschrift lühn die Begrundung - BR-Drs. 134/90, S.23 - auch den Militanschen Abschiemdiense an.

40 BR-Drs, I 14/90, S, 21.

41 Bizcr/Hammer/Pordesch/Roßnagel (En. 29), DuD 1990, S. 179. 
den Bundesbeauftragien für den Datenschutz unterstützen ( 3 Abs. I Nr. 5 ), also durch seine tcchnische Kompetenz dazu beitragen, das Reche auf informacionelle Selbstbestimmung besser zu schüizen. Zugleich soll es auf der anderen Seite das Bundeskriminalame, den Militärischen Abschirmdienst und den Verfassungsschutz unterstützen ( $\$ 3$ Abs. 1 $N_{\text {r. }}$ ). Sein technischer Sachverstand soll mithelfen, als riskant definierte Verhaitensweisen und Absichten effektiver auszuforschen - und damit das Reche auf informationelle Selbstbestimmung zu beschneiden. Das BSI kann aber nichr zugleich Tcchniksysteme encwickeln, die etwa die Vertraulichkeit von Kommunikation sicherstellr, und diejenigen unterstützen, die sie aufheben wollen, ohne eine der Aufgaben zu Gunsten der anderen zu verleczen.

Ebenfalis in den Bereich der inneren Sicherheit fallen die Unterstützungsaufgaben für dic staatliche Geheimhaltung, die das BSI von seinen Vorgängern ZfCH und ZSI übernimmt. Zum einen soll das BSI dic informationstechnischen Systeme oder Komponenten zulassen, die im Bereich des Bundes für dic Verarbcitung oder Übertragung von Verschiußsachen eingesetzr werden, und zurn anderen die für den Betrieb zugelassener Verschlüsselungsgerätc benötigen Schlüsseldaten herstellen $(\$ 3$ Abs. 1, Nr. 4).4z

Auch der zweite Schwerpunkt des Geseczes, die Unterstüzung der Staatssicherheic, wird jedoch dem selbstgesteckren Zweck, die "Verletzlichkeit der modernen Informationsgesellschafiu zu reduzieren, nicht gerecht. Denn ein Bundesamt darf nicht nur die Sicherheitsinteressen großer Institurionen oder der staatlichen Behörden verfolgen oder die Gewährleistung der inneren Sicherheit des Staates in den Vordergrund stellen. Damic würde die Bewältigung der Risiken, die für jeden einzelnen Bürger aus der allgegenwärrigen Anwendung der Informationstechnik erwachsen, diesem selbst überlassen. Er müßce der Durchsetzungsmacht »der Großenu crliegen, wenn nicht auch seine Ziele institutionalisiert gegenüber Exekurive und Wirtschaft vertrecen würden. Dic Gewährleistung von IT-Sicherheit muß daher vor allern darauf zielen, die Freibeitsgrundrechle der Bürger zu sichern. ${ }^{43}$ Der Gesezzentwurf sicht in dicsem Sinne jedoch nur die widersprüchliche Unterstürzung sowohl des Datenschutzbeauftragten als auch der Sicherheitsbehörden vor.

Für den Bürger bestehen drei zentralc Schurzziele: ${ }^{44}$ Er soll zum ersten als Nutzer der Informationstechnik für seine Bedürfnisse keinc Risiken in Kauf nehmen müssen. Zum zweiten ist eine gegen scine Interessen gerichtere Nutzung der Technik oder seiner Daten durch staatliche oder private Organisationcn zu verhindern. Drittens sind seine Rechte auf informationelless und kommunikative Selbstbestimmung ${ }^{6}$ sowie scin Fernmeldegcheimnis gegen das steigende Ausforschungsinteresse staatlicher Sicherheitsbehörden zu schützen:

Das Vorhaben, Solrwareprodukte hinsichtlich ihrer Sicherlieit und Verfügbarkeic zu bewerten und die Prüfergebnisse durch Zerifikate bekanntzumachen, kann die Markntansparenz im Sinne des Konsumentenschurzes verbessem. Nachhaitig verbessert würde die Verbrauchersicherbeit allerdings erst, wenn strenge Haftungsregelungen an die zertifizierten Eigenschaften geknüpft würden. ${ }^{37}$

Durch die immer größeren Sammlungen personenbezogener Dacen und die verbesserren Möglichkeiten der Übermitrlung und Auswercung wird es immer äringlicher,

42 Siehe un einzelnen die Begrundung, BR-Drs. 134/90, S. 21 .

4) Vgl. hicruu z. B. RoBnagel U. a. (Fn. 1), S. I 8 ff.

$44 \mathrm{Im}$ Geseczencwuro ust von diesen allan der Datenschuez berücksschugt.

4) 5. BVeriGE 65, , (42 If.).

46 S. hierzu Roßnagel, Das Reche auf (tele)kommunikauve Selbstbesummung, Kruischi Justuz 3/1990

47 S. huerzu nähce Bizer/Hammcr/Pordesch/Roßnagel (Fr. 12), S. 26 if. 
die Sicherheit der Betroffenen vor ungewünschten Informationstechnik-Anwendungen zu gewährleisten. Beispielsweise wird dic Transparenz des Kundenverhaltens durch die "Informatisierung der Kundenschnitrstelle" etwa bei elektronischen Bestellungen, Kreditanträgen oder der Kundenidentifizierung beim elektronischen Zahlungsverkehr stetig erhöht. Markecingstrategen versuchen mir den gewannenen Profilen, das Verbraucherverhalten zu beeinflussen. Über die im Gesetz vorgesehene rechnische Unterstützung des Datenschurzbeauftragıen hinaus müßte gerade das BSI die Entwicklung technischer Komponenten vorantreiben und sicherstellen, die - wie auf dem Wochenmarkt - anonyme Telecransaktionen ermöglichen.

Bürgersicherbcit kann in der Informationsgesellschaft nur gewährleister werden, rvenn der Bürger selbst in ausreichendem Maße seine Anonymität wahren und für ihn wichtige Nachrichten vor dem Zugriff Dritter verbergen kann, \& Protorypische Entwicklungen zeigen, daß dies mit Verschlüsselungssystemen gelingen kann. Voraussetzung für eine solche Verbesserung des Grundrechisschutzes mit Hilfe der Informationstechnık ist ein Verschlüsselungsverfahren, das für jedcrmann vcrfügbar ist und für das jeder die benörigten Schlüsscl für seine gewünschten Kommunikationspartner erhalten kann. Public-Key-Systcme erfüllen diese Bedingungen, denn die beiden Schlüssel zum Ver- und Entschlüsseln sind vcrschieden und ohne Zusatzwissen praktisch nicht gegenseitig ableitbar. Ein Schlüsscl des Paares wird dem Teilnehmer "privat * und geheim in einer Chipkarte zur Verfügung gestelit, während der andere in einem Directory, dem "Schlüssel-Telefonbuchu, veröffentlicht wird. Al Allerdings müssen die geheimen Schlüssel wirklich gehcim gehalten werden, sonst können Nachrichten manipulicrt, Ydencitäten vorgetäuscht oder verschlüsselte Nachrichten in den Klartext übersetze werden. ${ }^{50}$

\section{Betriebssicherheit statt Verletzlichkeit}

Aber nicht nur in der Beschränkung der Schutzobjekte verdient das Gesecz Kritik, sondern auch in der Begrenzung des Handlungsziels auf die Betriebssicherheit informationstechnischer Systeme." Die technische Sicherung von IuK-Systemen ist zwar ein wichtiger, aber kcineswegs ausreichender Beicrag. ${ }^{52}$ Indem das Geserz die Aufmerksamkeit allein auf die technische Verringerung der Wahrscheinlichkeit von Schadenseintritzen lenkt, berücksichtigt es nur die eine Hälfte des Problems der Verleczlichkeit dor Informacionsgesellschait. Um sie zu verringern, sind darüberhinaus jedoch die Schadenspotentiale, die durcla die Abhängigkeit von der Informationstechnik für dic Gescllschaft und den einzelnen Bürger geschaffen werden, die konkrcten Anwendungsbedingungen und die durch sie verursachten sozialen Folgen zu berücksichtigen. Zwar wurde auf entsprechende Kritik hin die Beratungspflicht des $₫ 3$ Abs. 1, Nr.7 um eine begrenzte Folgenabschätzung ergänzt. Doch kann eine Einzelfallberatung auf Anforderung, die neben der Betricbssicherheir

$4^{8}$ Vgl. hicras Pfilixmann/Pfitxmann/Waidner. Datenschutz garanticrende offene Kommanikationsnctze, InformatikSpektrum 1988, S. 118 if.

49 Zur Beschreibung beispiclsweise des Tcletruse Konxeptes der GMD vgl. die Autoren in GMID-Spicgel if 86 und $1 / 88$ sowne Hammer. Tele'TruseT: Verlearlichkelt und Verfassungsvenraglichkest esnes Konzeptes fur rechtssichere Transakuonen in der Iníarmationsgesellschaft, DuD 8/4 $98 \mathrm{~S}$, S. 391 if. so Zu Aspekten der Verleızlichkeıt s. Hammer (IFn. 49), S. 398 If.

s1 BR-Drs. 1 3 4/90. S. 1f., 9 ff.: $\$ 3$ Nr. 14 BSIG: Leibench (Fn. 15), S. 2.

s2 Vgl. zum folgenden ausführlicher Bizer/Hammer/Pordesch/Roßnagel (Fn, 1 2), S. z I ff. 
auch »mögliche Folgen fehlender oder unzureichender Sicherheitsvorkehrungen" berücksichtigt, die crforderliche Verletzlichkeitsanalyse nicht ersetzen.

Das Problem der Verleczlichkeit, nämlich die Möglichkeit großer Schäden für Einzelne oder dic Gesellschaft, entsteht vor allem dadurch, daß soziale Funkcionen von Menschen auf Informations- und Kommunikationssysteme übertragen werden. Informationsverarbeitung und Kommunikation werden dadurch vom Funktionieren einer Technik abhängig, auf die sich die Menschen verlassen. Im Verırauen auf die Technik erlı̈hen sie deren Leistungsfähigkeit - und damis zugleich das Schadensporencial. Durch diese Übertragung werden zudem Informationsverarbeitungsund Kommunikationsprozesse für Dritte zugänglich. Sie können diese leichrferrig oder mißbräuchlich ausforschen, manipulieren, unterbinden, beschädigen oder zerstören. Fehler und Manipulationen können so die Erfüllung der dem technisclien System übertragenen gesellschaftlichen Funktionen becinträ̈chrigen." ${ }^{\text {"3 }}$

Existentielle Voraussetzung für das Überleben in einer hochindustrialisierten Gesellschaft ist die Bereitstellung von Nahrung, Energiedienstleistungen, Klcidung, Fortbewegungs- und Zahlungsmirteln sowie anderen Gütern und Dienstleistungen zur Befriedigung der Grundbedürfnisse. Bcreirs heutc, jedenfalls aber in Zukunft werden gerade diese sozialen Funktionen ausmahmslos mit Hille von JuK-Technik gesteuert und sind von ihrem Funktionieren vollständig abhängig. Hohe Schadensporentiale können durch die Abhängigkeit von IuK-Systemen vor allem zu erwarten sein in den gesellschaftlichen Bereichen des Verkehrswesens, der Steucnung komplexer industrieller Prozesse, des Zahlungsverkehrs und der staatlichen und privaren Verwaltung.

Um diese Risiken zu verringern, genügt es nicht, lediglich entwicklungsbegleitend einheitliche Sicherheitsstandards herzustellen, informationstechnische Sicherheirskomponenten und -systeme zu erforschen und zu entwickeln sowie die Anwender und Hersteller von informationstechnischen Produkten zu beraten, die informarionsrechnische Enrwicklung aber als unbeeinflußbar hinzunehmen. Vielmehr ist es erforderlich, die Schadensporencialc, die durch dic stcigende Abhängigkeit von der Informationstechnik anwachsen, in den Blick zu bekommen und gestaltend zu beeinflussen. Notwendig ist, die Schadenspotenciale zu verringern, indem die Abhängigkeit der Gesellschaft von der IuK-Technik reduziert wird.

In den Blick zu fassen sind daher nicht nur die Risiken, die aus Sicherheirsmängein icchnischer Produkte entstehen, sondern auch die Risiken, die von den sozialen Bedingungen und Folgen der Informationstechnik-Nutzung und -Sicherung im betrieblichen und gesellschaftlichen Kontext hervorgeruien werden. Und als Risiken dürfen nicht nur die Ausfallkosten eincs defekten Tecloniksystems, der Verrat militärischer Gcheimnisse, die finanziellen Verluste durch Computerkriminalität oder verminderte Exportchancen verstanden werden. Als Risiken sind auch und vorwiegend dic Nachreile zu becrachren, die dem einzelnen Bürger sowie der Geselischaft durch den Ausfall der auf die IuK-Technik übertragenen sozialen Funkcionen (Verkehr, Energieversorgung, Prozeßstcucrung, Handel, Zahlungsverkehr usw.) entstehen. Außerdem sind die negativen Folgen zu begreifen, die sowohl durch die möglichen Schäden als auch durch die Sicherungsmaßnahmen zu ihrer Verhinderung für die Ausübung von Grundrechten und einen freien Prozeß politischer Willensbildung encstehen können.

Eine Gescllschaft, die - um mögliche Katastrophen auszuschließen - darauf ange-

83 S. zum Begriff der Verlerzlichkeı naher RoBnagel u. a. (Fn. 5). S. s If. son'ı zur Verleızlichkeı der im folgenden angesprochenen ernzelnen Anwendungsbercichc 81 ff., 92 ff., 98 ff. und igg ff. 
wiesen ist, Sicherheit gegenüber menschlicher Böswilligkeit zu gewährleisten, ist auf präventive gesellschaftliche Kontrolle angewiesen. Will sie im Interesse von Freiheir und Demokratic diesen Sicherungszwang vermeiden, muß sie ihre Abhängigkeit von Technik-Systemen und damit deren Schadenspotential reduzieren." Der Gesetzentwurf läßr jedoch in seincr Technikfixierung auch dic negativen sozialen Folgen unberücksichrigr, die der Sicherungszwang verursacht. Statc ihn zu verringern, strebt er an, ihn bestmöglich zu erfüllen.

\section{Vertane Chancen}

Das BSIG ist lediglich ein Organisacionsgesctz. Gercgelt werden Errichtung, Aufgaben und Befugnisse einer neuen Bundesobcrbehörde. Zugleich aber ist dieses Gesetz der erste Schritt zu einer rechtlichen Regulierung der Informationstechnik." Mit ihm werden Weichen gestellt, die weit über den eigentlichen Regelungsgegenstand eine neue Bundesbürokratic - hinausweisen - und zivar vor allem dadurch, daß vicles bewußi ungeregelt bleibt.

Mitcelbar verfolgt dieses Geserz das Ziel, die Sicherheit eincr Technik zu erhöhen. Dieses Zicl teilc es mit den vielen bestehenden Regelwerken des Technikrechts. Nun aber weist die IuK-Technik gegenüber allen bisher regulieren Techniken einige Besonderheiten auf, die es verbiesen, auf sie einfach die überkommenen Regulierungsmuster ordnungsrechclicher Gcfahrenabwehr zu übertragen. Sie ist, nur hinsichtlich der weniger wichtigen Elcmente greifbar, ïberwiegend jedoch immateriell. Sie entspricht nicht dem herkömmlichen Maschinenmodell der Technik, sondern besitzt Systemcharakter. Sie ist nicht auf einen Zweck festgelegt, sondern nahezu universell verwendbar. Daher ist sie nicht auf einen Anwendungsbereich beschränkt, sondern durchdringt fast allc Gesellschaftsbereiche. ${ }^{6}$ Aufgrund dieser Unterschiede ist es verständlich, daß das neuc Gesetz nicht die alten Regelungsmuster wählt. Aber die Verletzlichkeit der Gesellschaft allein mit Klassifizienung, Beratung und Zerifikaten verringern zu wollen, ist zu wenig. Vielmehr komme es darauf an, die Lern- und Reaksionsfähigkcit der Gesellschaft im Úmgang mit dieser Technik zu ermöglichen oder zu stärken. Hierzu könnte das neue Bundcsamt cinen sinnvollen Beitrag leisten - allerdings nur, wenn scine Aufgaben, Befugnisse und Organisationsstruktur dieser Zielsetzung angepaßt wären. ${ }^{17}$

Hierfür wäre das Bundesamt für die Sicherheit in der Inlomationstechnik als selbständige und unabhängıge Bundesoberbehörde zu errichten. Es darf nicht weisungsgebunden sein und daher nur ciner Rechtsaufsicht unterliegen. Die gesellschaftlich wichtige wie für die Ausübung der Kommunikationsgrundrechte sensible

\4 S. hucrzu näher Rolbnggel u, a. (Fn. f). S. i > If.

ss Die Kommunikationszechnik ist dagcgen, soivsil sic durch die Deutsche Bundespost Telekom angeboten wird, stark reglementien. Doch erstrecken sich diesc Regulicrungen nur avl das Nutzungsverhälnıs zwischan stantlichem Monopolanbiezer und Verbraucher, nichi jedoch auf dic Kontrolle und Bceinflussung der technischen Eniwicklung. Im Zuge der durch die P'osiserukturrcform eingeleneicn Deregulie-

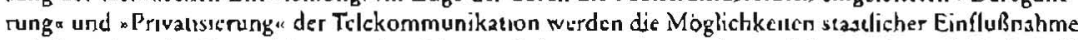
aul dic ecchrische En(wickluni noch starker zurückgenommen werden. - S. hicrzu knisch Roßnagel/ Wedde, Die Reform der Deusschen Bundespose im Liche des Demokracieprinzips, DVBI 1988, j6zlf.

56 RoBnage). Moglichkerten verfassungsvernraglicher Technikgestaltung, in: ders. (Hrsg.), Freihen im Grill. Informassonsgesellschaft und Grundgeselz, 1989, 177 il. jeweils mwN.

s) S. zum foigenden năher Bizer/Hammer/Pordusch/Roßnagel (Fn. I 2). S. 4 s lf, sawie dies. (Fn. 29), DuD 1990,184 f. 
Auf́abe der Verringerung der Verletzlichkeit der Geselischaft verträgt sich nicht mit ciner weisungsgebundenen Unterstellung unter den "Polizeiminister des Bundesa.

Als vorrangige Aufgabe zur Herstellung von Sichcrheit muß die Begrenzung des Schadenspotentzals angesehen werden. Denn nur uncer dieser Voraussetzung werden große Sicherungszwänge vermieden und kann auf die Einschränkung der Freiheitsgrundrechre von Bediencrn und Bürgern zur organisatorischen Sicherung der Technik verzichtet werden. Das Bundesamt sollte demnach dic Aufgabc haben, für die verschiedenen Anwendungen von Informationstechnik jeweils zu prüfen, welche Abhängigkeiten durch den Technikeinsatz entscehen. Im konkreten Fall sind dazu verschiedene Alternativen des Technikeinsatzes zu vergleichen und hinsichtlich der Folgen für die Gesellschafe und ihres Schadensporentials zu bewerten. Insbesondere ist zur Schadensbegrcnzung daraut zu achcen, daß Substitutionsmöglichkciten erhalten bleiben, die bei cinem Technikausfall zumindest einen "Notbctricb gewährleisten. Eine ähnliche Wirkung wird erreichs, wenn die Diversifikation von eingesetzten informationstechnischen Systemen garanticr ist.

Zu den Aufgaben des BSI sollte das Sammcln und Dokumentieren von Schadensfällen gehören. Nur dadurch kann das Bundesame über das notwendige Erfahrungswissen verfügen, mir dessen Hilfe die Wahrscheinlichkeic von Schadensfällen, deren Schadensausmaß sowie mögliche Gegenmaßnahmen ermittelt und eine Verringerung der Verletzlichkeit erreicht werden kann. Dazu benötigl das BSI ausreichende Informationen übcr die entwickelten und eingeserzten Systeme oder Komponenten der Informationstechnik sowie Kennenisse über Störfälle. Aus diesem Grund wärc eine Anzeigepflicht für das Herstellen, Errichten, Vertreiben und Betreiben von Informations- und Kommunikationssystemen in das BSIG aufzunehmen.

Das BSI sollte jährlich in einem zusammenfassenden Verletzlicbketsbericht an den Bundestag und die Bundesregicrung beschreiben, wie sich die Verleczlichkeit der Gesellschaft entwickelt hat. In diesem Bericht sollte das Bundesamt für die Sicherheit in der Informationstechnik insbesondere die Abhängigkeit der Gesellschaít von informationstcchnischen Systemen und das damit verbundene Schadenspotential darstellen und allen betroffenen gesellschaftlichen und scaatlichen Instanzen Vorschläge unterbreiten, wic sie durch Tcchnikgestaltung die Verleczlichkeit der Gesellschaft reduzicren können. Insbesondere indem das BSI mögliche Altcrnativen und Gegenmaßnahmen skizziert, könnte der Bericht das Problembewußtscin einer breicen Öffenclichkeic anregen.

Das BSI sollte, wo Bedarf dafür besteht, Modelivorhaben anstoßen und soziale Experimente unterstüzzen, die Alternativen zur Trendentwicklung in die Informationsgesellschar karscellen, denn das rechtzeitige Erkennen und Offenhalten von Alternativen zu technischen Entwicklungen trägt dazu bei, die Verletzlichkeit der Gcsellschaft zu verringern. In solchen Modellversuchen muß das Bundesamt immer auch versuchen, die Gegengewichte gegen die negativen Folgen einer Inímationsstrategie zu stärken. Für diese Alternativen sind die Verletzlichkeitsaspekte und die von ihnen ausgehenden sozialen, rechelichen und wirtschaftlichen Folgen abzu* schärzen.

Angesichcs der zunehmenden Bedeucung von Verschlüsselungssystemen sollten die Aufgaben der Zulassung informationstechnischer Systeme oder Komponenten auf den Bereich der Bundesbehörden bzw. auf Unternehmen, die im Rahmen von Aufträgen des Bundes tätig werden, beschränkı werden. Ebenso muß die Herstel-

(8 Sie sind daher in emem weiteren Diskussionszusammenhang zu eronern - s. hierzu z. B. Roßnsgel u. $a$. (Fn. 4). S. 286 if. 
lung der Schlüsseldaten auf die Verarbeitung oder Übertragung von Verschlußsachen des Bundes beschränkt bleiben. Die Entwicklung selbständiger vivilere Verschlüsselungsmechanismen darf nicht behindert werden und muß unabhängig vom BSI erfolgen.

Das BSI sollte stardessen einc unabhängige Erforschung, Entwicklung und Anwendung von Verschlüsselungssysremen fördern, denn Verschlüsselungssystemen kommt mit der zunehmenden Entwicklung von Informationstechniken eine zentrale Bedeucung für die Reduzierung der Verletzlichkeit und der Gewährleistung der Bürgersicherheit zu. Die Erforschung, Entwicklung und Anwendung von Verschlüsselungssystemen muß aber aus Gründen der Verleczlichkeit der Gesellschaft wie der Bürgersicherheit durch unabhängige staatsfreie Einrichtungen erfolgen, bei denen der Staat kcinen Einfluß auf den wissenschafdichen Erkenntnisprozeß und das Ergebnis hat. In diesem Zusammenhang könnte dem BSI die Aufgabe zufallen, die Normung für den Einsac\% von Public-Key-Systemen zu fördern, Fachkompetenz für die öffentliche Diskussion der Vertrauenswürdigkeit des Verfahrens bereirzustellen und die verwendeten Sysreme zu validieren.

Informations- und Kommunikationstechnik findet in vielen gesellschafelichen Bereichen Anwendung, für die cine Sicherheitsbewertung in Genehmigungs., Zulassungs- und Planungsverfahren erforderlich ist. Soweir die Sicherheir in der Informationstechnik berühn ist, sollte das Bundesamt Empfeblungen zur Reduzierung der Verlerzlichkeit abgeben können. Darïberhinaus sollte das BSI in Einzelfällen Anordnungen für dic technische oder organisatorische Gestaltung der informationstechnischen Systeme oder Komponenten treffen können, wenn die Auswirkungen auf die Verletzlichkeit der Gesellschaft in einem unvertretbaren Ausmaß vernachlässigt werden. Die Verietzlichkeit der Gesellschaft erfordert eigentlich weitergehende Genehmigungs- und Zulassungsverfahren nach Maßgabe prävenciver Kontrolle. In solchen Verfahren wären allerdings nicht nur Verleczlichkeitsprüfungen durchzuführen, sondern es wären vielmehr weircre Gesichtspunkte wie Dacenschutz, Verfassungsverträglichkeic, Arbeirsschucz, Verbraucherschucz zu berücksichrigen. ${ }^{8}$

Die Bundesregierung hat Anregungen dieser Art nicht aufgegriffen. Whr Gesetzentwurf ist vielmehr von einer seltsamen Mischung geisciger Ursprünge gepragt: Dic Wurzeln dieses Gesetzes sind zum einen in dem Bemühen der Nachrichtendienstler zu suchen, das sraacliche Geheimnis auch in das Zeizalier der Informarionsgesellschafu zu retten. Auch angesichts verblüffender Hacker-Coups, zerstörerischer Compurer-Viren und kompromittierender Abstrahlung soll es künftig möglich sein, Verschlußsachen so geheim zu bearbeiten, wie dies für Geheimniskrämer mitcels Papier und Tinte möglich war. Die zweite Wurzel ist die Nachfrage ssicherer" Computer und Telekommunikation insbesondere im miliärischen Bereich und der internationale Wettbewerb um diese lukrativen Aufräge. Hierfür sind Standards, Produktprüfungen und Zertifikate notwendig, die die geforderte Sicherheit nachweisen. Nun soll die übrige Verwaltung von den Errungenschaften sicherer Gcheimnisbearbeitung und -übertragung und die übrige Wirtschaft von der Zertifizierung ssicherer \& IuK-Produkce profitieren. Schließlich atmer das Gesetz den Geist des Wirtschafesliberalismus und ist gepräge von dem Glauben an die Allmacht des Marktes. Die technische Entwicklung selbst bleibr außerhalb des Blickfeldes. Ein secuernder oder auch nur korrigicrender Eingriff aus Sicherheitsgesichtspunkren ist nicht vorgesehen. Weder im staatlichen noch im nicht-staatlichen Bereich soll dic zunchmonde Abhängigkeir der Gesellschaft und das Entstehen großer Schadenspotentiale in irgendeiner Weise beeinflußt noch sollen gar gezielt sozio-technische Alternativen entwickelt werden. Kein Technikgesetz hatte bisher solche Eltern: Nachrichtendicnste, Protektionismus und Wirtschaftsliberalismus. 
Der Gesetzentwurf erscheint als ein halbherziger Versuch der Bundesregierung, einem von ihr mitverursachten Dilemma entgchen zu wollen. Auf der cinen Seire forciert sie den Wettlauf in die "Informationsgesellschaftu, auf der anderen Seite muß sic aber die Sicherheirsgefahren und Sicherungszwänge crkennen, die sie darnit setzt. Diese Risiken gefährden nun die Akzeptanz des cingeschlagenen Weges in die »Informationsgesellschaft a und zwingen zum Handeln. Um aber ihre Förderpolitik nicht ändern zu müssen, versucht dic Bundesregierung, diese Risiken so zu definieren, daß sie durch die Errichcung cines Bundesamces und seiner im wesentlichen auf die Verbessenumgen der Sicherheitstechnik begrenzten Aufgabenstellung lösbar erscheinen. 